\title{
Utilização de fonte taninífera na dieta de caprinos como alternativa de controle parasitário
}

\author{
The use of tanniferous plant in the goat diet as alternative to \\ parasite control
}

\author{
Eduardo Shalders ${ }^{1}$; Surama Freitas Zanini ${ }^{2 *}$; Dyeime Ribeiro Sousa ${ }^{3}$; \\ Renata Cogo Clipes; ${ }^{4}$ Graziela Barioni ${ }^{5}$; Barbara Rauta Avelar ${ }^{3}$; \\ Jamili Maria Mussi Suhet ${ }^{6}$
}

Resumo

O presente trabalho avaliou a eficácia da torta de pimenta rosa (TPR) na dieta de caprinos leiteiros nas fases de cria e recria, como agente antiparasitário frente ao controle da coccidiose com ionóforo, monensina sódica. Foram utilizadas 18 cabras, da raça Saanen, distribuídos em delineamento inteiramente casualizado em grupos de três tratamentos e seis repetições, constituídos pelos grupos experimentais: grupo 1 - controle sem adição de ionóforo ou de TPR; grupo 2 - suplementado com $30 \%$ de TPR; grupo 3 - controle positivo adição de monensina sódica. Observou-se efeito significativo dos tratamentos sobre o consumo voluntário animal, tanto na fase de cria quanto na de recria, sendo que os animais suplementados com TPR tiveram os menores valores de consumo comparado com os demais grupos $(\mathrm{P} \leq 0,05)$, mas sem interferir negativamente no peso corporal. Em geral, tanto na fase de cria quanto de recria, o consumo voluntário entre os grupos controle negativo e controle positivo, não diferiram entre si $(\mathrm{P}>0,05)$. Na fase de recria constatou-se que houve efeito dos tratamentos sobre o peso corporal $(\mathrm{P} \leq 0,05)$, a partir da sétima semana de experimento. Os animais alimentados com TPR tiveram um peso corporal superior ao controle negativo $(\mathrm{P} \leq 0,05)$ e similar aos tratados com a monensina sódica $(\mathrm{P}>0,05)$. A contagem de oocistos por grama de fezes foi menor nos animais tratados com TPR diferindo significativamente do controle negativo $(\mathrm{P} \leq 0,05)$. Em relação à contagem de ovos por grama de fezes não houve efeito significativo dos tratamentos $(\mathrm{P}>0,05)$. Verificou-se que o TPR foi eficaz no controle dos protozoários da família Eimeriidae, com potencial para serem utilizadas em programas alternativos de controle da eimeriose caprina. Concomitantemente, também maximizou o desempenho animal conferindo maior peso corporal mesmo com a redução de consumo voluntário.

Palavras-chave: Pequeno ruminante, pimenta rosa, monensina sódica, taninos, eimeriose

\footnotetext{
${ }^{1}$ Discente do Programa de Pós-Graduação em Ciências Veterinárias, Universidade Federal do Espírito Santo, UFES, Campus Alegre, ES. E-mail: dududshalders@hotmail.com

${ }^{2}$ Prof. Associado II da Universidade Federal do Espírito Santo, UFES, Dept ${ }^{\circ}$ de Medicina Veterinária, Campus Alegre, ES. E-mail: smzanini@gmail.com

${ }^{3}$ Discentes do Programa de Pós-Graduação em Ciências Veterinárias, Universidade Federal do Espírito Santo, UFES, Campus Alegre, ES. E-mail: dyeimester@gmail.com; binha_rauta@hotmail.com

${ }^{4}$ Prof. do Instituto Federal do Espírito Santo, IFES,Campus de Alegre. ES. E-mail: rclipes@hotmail.com

${ }^{5}$ Prof. Adjunto da Universidade Federal do Espírito Santo, UFES, Dept ${ }^{\circ}$ de Medicina Veterinária, Campus Alegre, ES. E-mail: graziela.barioni@terra.com.br

${ }^{6}$ Discente do Programa de Pós-Graduação em Ciência Animal, Universidade Federal de Minas Gerais, UFMG, Belo Horizonte, MG. E-mail: jamilisuhet@hotmail.com

* Autor para correspondência
} 


\begin{abstract}
This study evaluated the effectiveness of pink pepper meal supplementation (PPM) in the dairy goats diet as an antiparasitic agent compared with ionophore, the monensin sodium. A total of 18 female Saanen goats, were distributed in a completely randomized in groups of three treatments and six replications, consisting of the experimental groups: negative control (did not receive anticoccidial agent or PPS), group 1 (supplemented with $30 \%$ of PPM) and group 2 (supplemented with anticoccidial agent - monensin sodium). There was a significant effect of treatments on voluntary feed intake on growing phases $(\mathrm{P} \leq 0.05)$. Furthermore, the animals treated with PPM had the lowest food consumption compared with the other treatments $(\mathrm{P} \leq 0.05)$, but without negative effect on live body weight. In general, at both phases of rearing, it was not detected significant difference between the voluntary feed intake of control group and with monensin sodium supplementation $(\mathrm{P}>0.05)$, but the food consumption in the control group did not result on increase of live body weight probably due to sanitary challenges such as gastrointestinal parasites. The opposite was observed in animals treated with monensin sodium that had the highest body weight $(\mathrm{P} \leq 0.05)$ that did not differ from animals fed with pink pepper ( $\mathrm{P}$ $>0.05)$. By the way, in the growth phase our results revealed significantly effect of treatments on live body weight $(\mathrm{P} \leq 0.05)$. Animals fed with pink pepper supplementation had a greater live body weight compared with the control $(\mathrm{P} \leq 0.05)$ and similar to those supplemented with the monensin sodium $(\mathrm{P}>0.05)$. The number of oocysts per gram of faeces was lower in animals treated with PPS that differed significantly from control $(\mathrm{P} \leq 0.05)$. Regarding the count number of eggs per gram of faeces OPG, it was not detected significant effect of treatments $(\mathrm{P}>0.05)$. It was found that the pink pepper supplementation was effective in the control of gastrointestinal parasites of goats, mainly on protozoa family Eimeriidae, revealing potential for use in alternative parasite control programs. Concomitantly also it was showed maximizing animal performance by high live body weight even with the reduction of voluntary feed intake.
\end{abstract}

Key words: Small ruminant, pink pepper, tannin, antiparasitic agent, eimeiriosis

\section{Introdução}

$\mathrm{O}$ aspecto sanitário têm se constituído num sério obstáculo à caprinocultura, com ênfase para os parasitos gastrintestinais, que ao acarretar infecções clínicas promovem retardo no ganho de peso, gastos com tratamentos e, em casos extremos, mortalidade de animais, especialmente animais jovens e fêmeas no periparto, fatores esses que limitam exploração econômica (FORBES; CUTLER; RICE, 2002; LIMA; AHID; SIMPLÍCIO, 2009).

Os caprinos com aptidão leiteira são bastante susceptíveis as infecções parasitárias, principalmente as eimerioses, sendo os animais jovens os mais susceptíveis devido à baixa imunidade ao enteroparasita e os adultos os mais resistentes, sendo considerados portadores e disseminadores da doença no rebanho (COSTA; SIMÕES; RIET-CORREA, 2009; FREITAS et al., 2005; LEVINE, 1985). A eimeriose se manifesta por transtornos intestinais, limitando a produtividade e exigindo medidas de controle urgentes, uma vez que determinam prejuízos econômicos que refletem, no aumento de índice de mortalidade entre animais jovens (VIEIRA, 2008).

O controle parasitário realizado com alguns antihelmínticos sintéticos tem promovido ocorrência de populações resistentes as drogas disponíveis atualmente (MAX et al., 2009), a crescente preocupação de resíduos na cadeia alimentar e com a poluição do ambiente (ATHANASIADOU; HOUDIJK; KYRIAZAKIS, 2008; GRAMINHA et al., 2005), desta forma, torna-se imprescindível a busca por medidas alternativas de controle (CEZAR; CATTO; BIANCHIN, 2008).

O uso de plantas taniníferas na dieta animal pode influenciar positivamente a sanidade de pequenos ruminantes (OLIVEIRA et al., 2011) pela diminuição da degradação das proteínas no rúmen, com aumento da quantidade de proteína disponível no intestino delgado, com a eliminação 
de parasitas e com a diminuição do timpanismo espumoso (VASTA et al., 2009). A eficácia dessas plantas são, geralmente, sobre redução no número médio de ovos por grama de fezes (OPG) que foram observadas por Almeida et al. (2007), Max et al. (2009), Minho et al. (2008a), Parra et al. (2011) e Silva et al. (2010).

A ação dos taninos condensados tem sido relacionada com redução da fecundidade dos nematóides (FALBO et al., 2008; HOSTE et al., 2006), redução da eclosão dos ovos e com a redução do desenvolvimento das larvas, uma vez que não ocorre absorção deste composto no trato gastrintestinal são eliminados pelas fezes e, assim atua na descontaminação do pasto (HECKENDORN et al., 2007; MUPEYO et al., 2011).

Os ionóforos em doses baixas podem não controlar a eimeriose (LIMA; AHID; SIMPLICIO, 2009; VIEIRA et al., 2005), vale ressaltar, que a depender da dose, os ionóforos podem levar a intoxicação e morte dos animais (AFONSO et al., 2000; AGAOGLU et al., 2002; SANTA-ROSA; PINHEIRO, 1996).

Pesquisas utilizando plantas e resíduos agrícolas com atividade anti-helmíntica têm possibilitado a comprovação de seu efeito no controle de parasitoses (NOGUEIRA et al., 2012; VIEIRA, 2008; VIEIRA et al., 1999).

A pimenta-rosa (Schinus terebinthifolius Raddi)é o fruto da aroeira vermelha que é uma espécie nativa, pertencente à família Anacardiaceae, que contêm flavonóides, esteróides, triterpenos, antraquinonas, saponinas e fenóis como taninos (LIMA et al., 2006), sendo considerada um produto agrícola com potencial de utilização na alimentação animal (ZANINI et al., 2009a; 2009b; GONÇALVES et al., 2012). Apresenta atividade anti-inflamatória (GAZZANEO; LUCENA; ALBUQUERQUE, 2005) e antimicrobiana (MARTINEZ; GONZALES; BETNCOURT, 1996).

Com isso, objetivou-se avaliar a eficácia da torta de pimenta rosa (Schinus terebinthifolius Raddi) na dieta de caprinos leiteiros nas fases de cria e recria, como agente antiparasitário frente ao tratamento com ionóforo, com monensina sódica.

\section{Material e Métodos}

O experimento foi realizado nas instalações para caprinos do Rancho Visoleta Capril SH, localizado no município de Itapemirim-ES, nas coordenadas geográficas, latitude 2053'51'S, longitude 4052’38” W e altitude de nove metros. O clima da região é quente e úmido (tropical), segundo a classificação de Köppen-Geiger descrita por Kottek et al. (2006).

Foram utilizadas dezoito cabras jovens da raça Saanen, distribuídas em delineamento inteiramente casualizado em grupos de três tratamentos e seis repetições, constituídos pelos grupos experimentais: grupo 1 - controle sem adição de ionóforo ou de TPR; grupo 2 - suplementado com $30 \%$ de TPR; grupo 3 - controle positivo adição de monensina sódica. A monensina sódica foi o agente anticoccidiano adicionado ao concentrado na dosagem de 30 gramas por tonelada (STOCK; MADER, 1997).

Visando homogeneização dos tratamentos, os animais foram distribuídos aleatoriamente conforme variação de peso e idade. Portanto, para a formação dos grupos experimentais havia dentro de cada tratamento duas categorias animais: fêmeas de 50 a 70 dias de idade e peso vivo (PV) médio de $12 \mathrm{~kg}$ e fêmeas de 120 a 150 dias de idade e PV médio de $20 \mathrm{~kg}$, sendo consideradas nas fases de cria e recria, respectivamente, segundo Vieira et al. (2004).

Os animais foram mantidos em regime de confinamento, em baias individuais, medindo 0,50 x 1,2 metros, em galpão coberto, com piso ripado, providas de comedouro e bebedouro, recebendo água à vontade e alimento volumoso duas vezes ao dia, totalizando em média $4,0 \%$ do $\mathrm{PV}$, em quantidade suficiente para permitir uma sobra entre $10 \%$ e $15 \%$ do oferecido. Inicialmente os animais foram submetidos a um período de 14 dias de 
adaptação às instalações, ao manejo, à dieta e ao ajuste do consumo voluntário.

$\mathrm{Na}$ ração teste (grupo 2) a torta pimenta rosa substituiu 30\% do milho, como apresentado na Tabela 1. As rações foram isoproteicas com cerca de $20 \%$ de proteína bruta. Os ingredientes foram misturados em um equipamento com capacidade para $500 \mathrm{~kg}$. A proporção de volumoso foi $70 \%$ e concentrado foi de $30 \%$, pesados em balança eletrônica e misturados manualmente nos cochos sendo que as quantidades oferecidas foram ajustadas semanalmente, por ocasião da pesagem individual dos animais. As sobras de alimentos de cada baia foram quantificadas diariamente, possibilitando o cálculo posterior do consumo e ajuste da quantidade de alimento a ser fornecida de maneira a proporcionar sobras diárias de aproximadamente $10 \%$. O período experimental teve duração de nove semanas, sendo que não houve tratamento anti-helmíntico, antes e durante o período experimental.

Tabela 1. Composição dos ingredientes nas rações fornecidas a caprinos, de acordo com o grupo experimental

\begin{tabular}{|c|c|c|c|}
\hline \multirow{2}{*}{ Ingredientes } & \multicolumn{3}{|c|}{ Tratamentos } \\
\hline & Grupo $1^{1}$ & Grupo $2^{2}$ & Grupo $3^{3}$ \\
\hline Milho moído & 380,00 & 266,00 & 380,00 \\
\hline Farelo de soja & 146,00 & 146,00 & 146,00 \\
\hline Farelo de trigo & 206,00 & 20,00 & 20,00 \\
\hline Torta de pimenta rosa & ---- & 300,00 & ------ \\
\hline Calcário & 11,30 & 11,30 & 11,30 \\
\hline Fosfato bicálcico & 3,80 & 3,80 & 3,80 \\
\hline Sal & 3,80 & 3,80 & 3,80 \\
\hline Suplemento mineral ${ }^{4}$ & 0,380 & 0,380 & 0,380 \\
\hline Suplemento vitamínico ${ }^{5}$ & 0,150 & 0,150 & 0,150 \\
\hline
\end{tabular}

${ }^{1}$ Grupo 1: Controle negativo - concentrado sem adição de agente anticoccidiano; ${ }^{2}$ Grupo 2 : suplementado com $30 \%$ de torta de pimenta rosa; ${ }^{3} \mathrm{Grupo} 3$ : controle positivo - concentrado contendo agente anticoccidiano, monensina sódica na dose de 30 gramas por tonelada; ${ }^{4} \mathrm{Ca} 22 \%$, P 5,5\%; Mg 3,5\%; S 2,2\%, Cl 10,55\%; Na 7\%; Mn 1500 mg/kg; Fe 500 mg/kg; Zn 1550 mg/kg; Cu 440 $\mathrm{mg} / \mathrm{kg}$; Co $50 \mathrm{mg} / \mathrm{kg}$; I $40 \mathrm{mg} / \mathrm{kg}$; Se $20 \mathrm{mg} / \mathrm{kg} ;{ }^{5}$ Vitamina A 135.000,00 UI, D3 68.000,00 UI, Vitamina E 450,00UI.

Fonte: Elaboração dos autores.

Foi determinada a composição químicabromatológica da torta de pimenta rosa, obtido de frutos maduros sem a extração do óleo, de acordo com a metodologia de Silva (1998). Os teores de taninos do farelo e das rações experimentais foram determinados com base nos trabalhos de Dawra, Makkar e Singh (1988), Inoue e Hagerman (1988), Makkar et al. (1993) e Porter, Hrstich e Chan (1985). Os resultados da avaliação química-bromatológica e do conteúdo de compostos fenólicos presentes na TPR encontram-se na Tabela 2.

Os parâmetros avaliados foram: 1 - consumo animal, no qual os animais foram alimentados individualmente as $6 \mathrm{~h}$ e $18 \mathrm{~h}$, recebendo a ração concentrada misturada com a silagem de milho verde picado, sendo o consumo voluntário calculado pela diferença entre o fornecido e as sobras, 2 - peso animal, onde a pesagem individual dos animais foi executada em intervalos de 07 dias até o fim do experimento, mediante jejum.

Além disso, amostras de fezes foram coletadas, durante o período experimental, em intervalos de sete dias, direto da ampola retal em sacos plásticos de $03 \times 12 \mathrm{~cm}$, devidamente lubrificados com glicerina. As amostras foram identificadas, acondicionadas em caixas de isopor com gelo e encaminhadas ao laboratório de Doenças Parasitarias do Centro de Ciências Agrárias da 
Universidade Federal do Espírito Santo (CCAUFES) para o processamento. Os exames foram realizados de acordo com as técnicas descritas por Gordon e Whitlock (1939). Os resultados da contagem do número de ovos por grama de fezes (OPG) foram transformados para $\log _{10}(\mathrm{x}+10)$, visando à uniformização da variância das médias.

Os resultados dos parâmetros avaliados foram submetidos à análise de variância com teste de médias, Student-Newman-Keuls (SNK), considerando o nível de significância de $5 \%$ (p $\leq$ $0,05)$.

Tabela 2. Determinação dos componentes químicobromatológica e quantificação de compostos fenólicos, presentes na torta de pimenta rosa.

\begin{tabular}{lc}
\hline Composição & Resultados $(\mathrm{g} / 100 \mathrm{~g})$ \\
\hline Matéria Seca & 87,10 \\
Umidade & 12,90 \\
Proteína Bruta & 7,10 \\
Extrato Etéreo & 9,74 \\
Matéria Mineral & 3,53 \\
Cálcio & 1,12 \\
Fósforo & 0,33 \\
Fibra Bruta & 21,35 \\
Fibra em detergente ácido (FDA) & 22,94 \\
Fibra em detergente neutro (FDN) & 45,05 \\
Fenós totais ${ }^{1 *}$ & 2,864 \\
Taninos totais* & 2,494 \\
Taninos condensados ${ }^{2 *}$ & 0,187 \\
\hline
\end{tabular}

${ }^{1}$ Valores expressos em equivalente grama de ácido tânico por $100 \mathrm{~g}$ de MS;

${ }^{2}$ Valores expressos em equivalente grama de leucocianidina por $100 \mathrm{~g}$ de MS.

*compostos fenólicos

Fonte: Elaboração dos autores.

\section{Resultados e Discussão}

Houve efeito significativo dos tratamentos sobre o consumo voluntário animal, tanto na fase de cria quanto na de recria, sendo que os animais que receberam TPR tiveram baixo consumo (Figura 1), quando comparados com os demais grupos ( $\mathrm{P} \leq$ 0,05 ) sem comprometer o peso corporal (Figura 2). Observou-se que os animais alimentados com TPR tiveram peso corporal superior ao controle negativo $(\mathrm{P} \leq 0,05)$ e similar, aos tratados com monensina sódica $(\mathrm{P}>0,05)$.

Essa alteração no consumo e peso relacionados a suplementação com TPR pode ser explicada pelas concentrações de taninos condensados na dieta, visto que esses podem formar um complexo taninos condensados/proteína, que ao entrar em contato com a saliva pode causar uma sensação de adstringência, diminuindo a palatabilidade e o consumo de alimentos (OTERO; HIDALGO, 2004). Apesar disso, a proteína complexada/taninos condensados pode proteger as proteínas da degradação ruminal, aumentando sua disponibilidade no intestino delgado, melhorando assim a absorção dos aminoácidos (ATHANASIADOU et al., 2001). No presente estudo observa-se a ação benéfica dos taninos em moderadas concentrações, revelado pelo peso corporal das fêmeas nas fases de cria e recria (Figura 2). De acordo com Barry, Mcneill e Mcnabb (2001) baixas concentrações de taninos na dieta de ruminantes aumentaram a absorção de proteínas.

Em geral, tanto na fase de cria quanto de recria, o consumo voluntário do grupo controle negativo e controle positivo, não diferiu significativamente $(\mathrm{P}>0,05)$, entretanto o consumo dos animais do grupo controle negativo não foi traduzido em peso corporal, provavelmente, devido aos desafios sanitários do ambiente, como por exemplo, as parasitoses gastrintestinais. $O$ contrário foi observado com os animais tratados com monensina sódica que tiveram elevado peso corporal $(\mathrm{P} \leq$ $0,05)$. 
Figura 1. (A) Avaliação da eficácia dos tratamentos antiparasitários sobre o consumo voluntário de caprinos na fase de cria. (B) Avaliação da eficácia de tratamentos antiparasitários sobre o consumo voluntário de caprinos na fase de recria. Grupo1: Controle negativo, sem adição do ionóforo ou de torta de pimenta rosa, Grupo 2: suplementado com TPR e Grupo 3: controle positivo recebeu monensina sódica na dose de 30 gramas por tonelada). * letras iguais seguida nas colunas os tratamentos não diferem entre si.
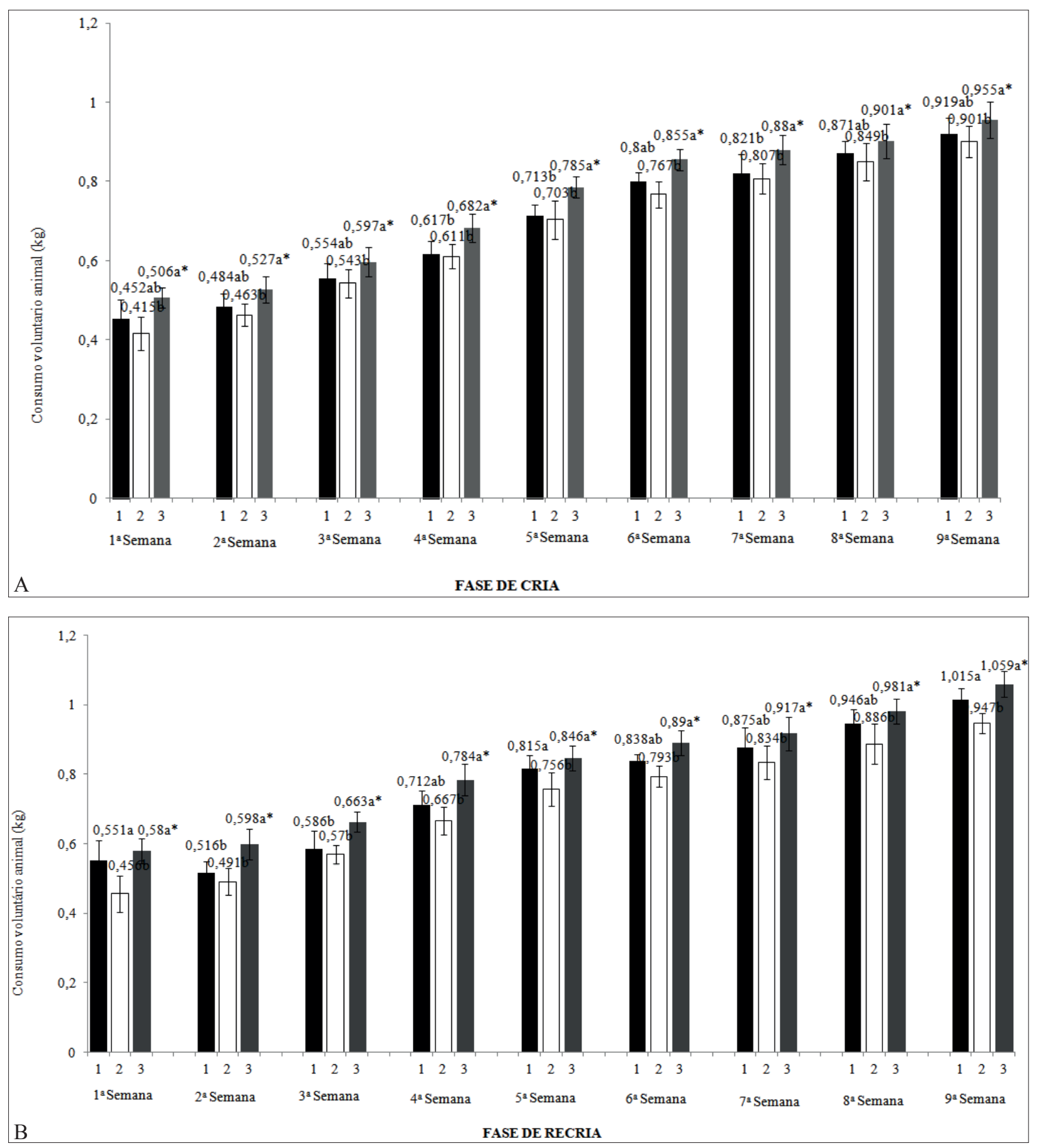

Fonte: Elaboração dos autores. 
Figura 2. (A) Avaliação da eficácia dos tratamentos antiparasitários sobre o peso corporal de caprinos na fase de cria. (B). Avaliação da eficácia de tratamentos antiparasitários sobre o consumo voluntário de caprinos na fase de recria. Grupo1: Controle negativo, sem adição do ionóforo ou de torta de pimenta rosa, Grupo 2: suplementado com TPR e Grupo 3: controle positivo recebeu monensina sódica na dose de 30 gramas por tonelada). * Colunas seguidas de letras iguais e/ou sem identificação não diferem entre si.

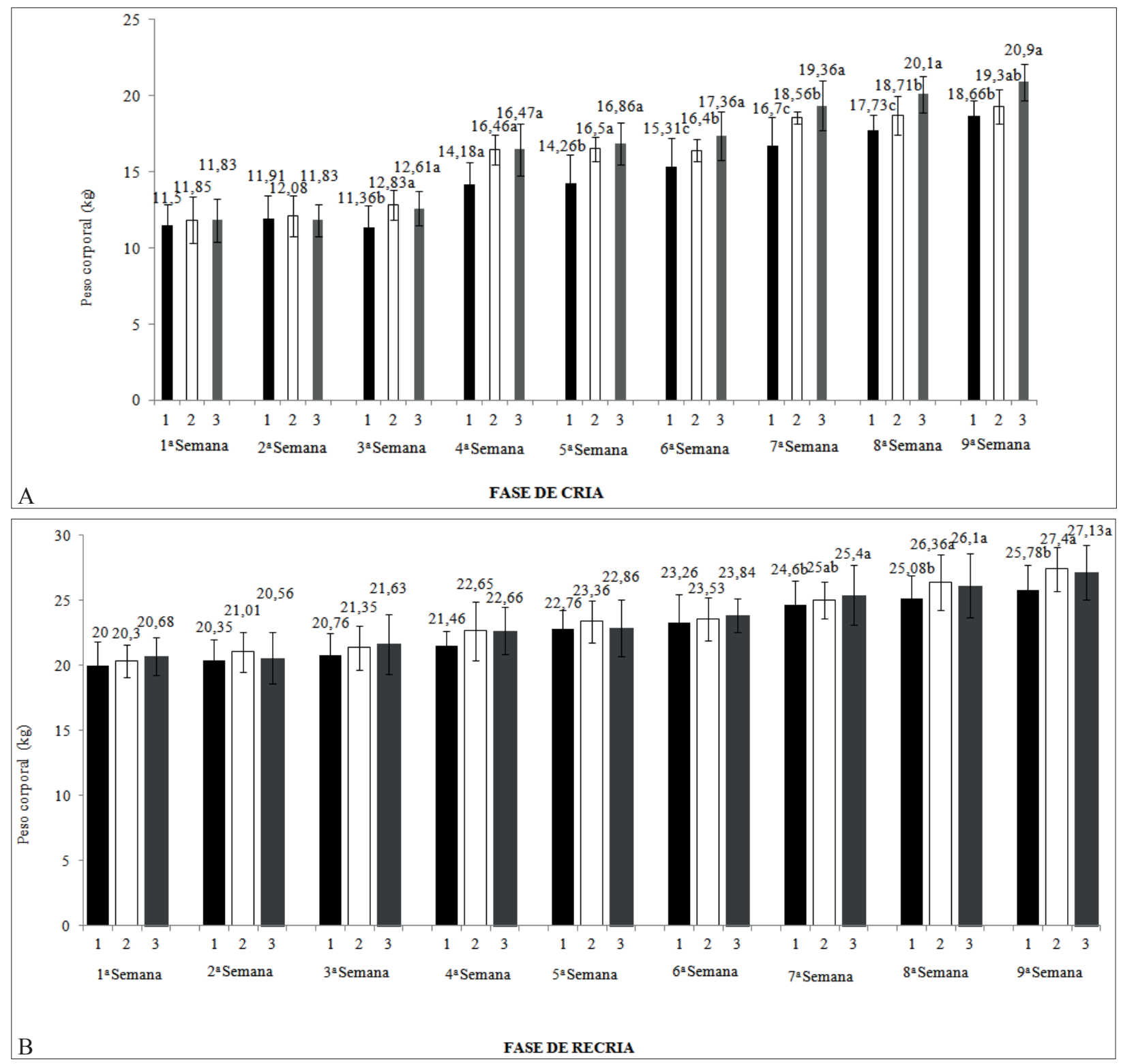

Fonte: Elaboração dos autores.

Segundo Costa, Simões e Riet-Correa (2009) animais jovens são mais afetados por enfermidades parasitárias devido à falta de imunidade contra os enteroparasitas, sendo necessário tratamento preventivo para evitar mortalidade. Vieira et al. (2004) avaliaram a salinomicina no controle da eimeriose caprina, observaram que na fase de cria, não houve diferença no ganho médio de peso, diferindo do presente estudo, mas corroborando com os estudos de Oliveira et al. (1997).

Os efeitos dos ionóforos sobre o ganho de peso pode advir por redução da degradação das 
proteínas no rúmen, permitindo a sua digestão e absorção no intestino delgado; pelo aumento de aminoácidos glicogênicos na corrente sanguínea oriundos do intestino delgado; ou ainda de forma indireta ao atuar sobre a digestibilidade da fibra, a qual diminui a concentração de lactato no rúmen, limitando assim, a queda do $\mathrm{pH}$ nesse ambiente, propiciando desse modo, melhores condições para o desenvolvimento de bactérias celulolíticas (BERGEN; BATES, 1984; RUSSEL; STROBEL, 1988).

Nas dietas que contém níveis elevados de carboidratos rapidamente fermentáveis, os ionóforos geralmente deprimem o consumo de alimento, porém o ganho de peso não é diminuído e a conversão alimentar é melhorada. Quando bovinos são alimentados com dietas contendo consideráveis quantidades de forragem, os ionóforos não reduzem o consumo, propiciando ainda aumento no ganho de peso dos animais (NAGARAJA et al., 1981).

Os resultados na fase de recria revelaram que houve efeito dos tratamentos sobre o peso corporal $(\mathrm{P} \leq 0,05)$, a partir da sétima semana de experimento (Figura 2 B). Os animais alimentados com TPR tiveram um peso corporal superior ao controle $(\mathrm{P} \leq$ $0,05)$ e similar aos animais tratados com monensina sódica $(\mathrm{P}>0,05)$.

Vieira et al. (2005) observaram efeito significativo $(\mathrm{P}<0,05)$ no ganho de peso para animais da raça Anglo-nubiana tratados com monensina sódica, na dose de $0,5 \mathrm{mg} / \mathrm{kg}$ de peso vivo, em relação ao grupo controle. Lima, Ahid e Simplício (2009) também observaram aumento de peso em animais que receberam ionóforos (salinomicina e monensina sódica) em relação ao controle, portanto, os resultados desse trabalho corroboram com esses autores. Contudo, Simplício et al. (2011) não observaram ganho de peso na fase de recria.

Os resultados encontrados neste experimento revelaram o efeito dos tratamentos sobre a contagem de oocistos por grama de fezes (OOPG) de protozoários da família Eimeriidae $(\mathrm{P} \leq 0,05)$.

Os animais tratados com TPR tiveram a menor contagem de OOPG diferindo significativamente ( $\mathrm{P}$ $\leq 0,05$ ) do grupo controle negativo (Figura 3). O tratamento com monensina sódica (grupo3) também resultou em redução da OOPG diferindo do grupo 1, mas inferior ao alcançado pelos animais tratados com TPR $(\mathrm{P} \leq 0,05)$.

Redução nas contagens de OOPG e melhoria no ganho de peso de animais tratados continuamente com antibióticos ionóforos foram descritas em caprinos nas fases de cria (OLIVEIRA et al., 1997; VIEIRA et al., 2005) e de recria (BARROS; SANTOS; FERREIRA, 1999; VIEIRA et al., 2004) resultados confirmados no presente trabalho.

Em relação à contagem do $\mathrm{OPG}$ não houve diferença entre os tratamentos $(P>0,05)$ em ambas as fases, resultados que corroboram com os estudos de Nascimento et al. (2009). Todavia, a avaliação da eficácia de plantas taniníferas in natura, sobre a redução do número médio de $\mathrm{OPG}$ foram observadas por Silva et al. (2010) e Parra et al. (2011). Essa diminuição pode provir da diminuição da carga parasitária, ou da redução da fecundidade das fêmeas de nematóides (HOSTE et al., 2005; JOSHI et al., 2011; MAX et al., 2009; MINHO et al., 2008b; SHAIK et al., 2006; TERRIL et al., 2007).

A atividade anti-helmíntica dos taninos condensados, presentes nas plantas taniníferas, já foi avaliada in vitro, por diversos autores (ALEMÁN et al., 2011; GOMES et al., 2010; OLIVEIRA et al., 2010) por ter como vantagens baixo custo, rapidez dos resultados, permitir a triagem de uma grande quantidade de plantas e possibilidade de avaliar substâncias isoladas sem interferência de outros compostos (GITHIORI; ATHANASIADOU; THAMSBORG, 2006).

Min e Hart (2003) apresentaram resultados demonstrando o efeito dos taninos condensados, frente a uma resposta imune ao parasitismo em animais, quando comparados com um controle de 
forragem com baixo teor do mesmo. A essa dieta também foi associada uma redução no número de
Haemonchus e Trichostrogylus no trato digetório dos animais (MIN et al., 2003; 2005).

Figura 3 Avaliação da eficácia dos tratamentos antiparasitários sobre a contagem total de oocistos de caprinos na fase de cria e recria. Avaliação da eficácia de tratamentos antiparasitários sobre o consumo voluntário de caprinos na fase de recria. Grupo1: Controle negativo, sem adição do ionóforo ou de torta de pimenta rosa, Grupo 2: suplementado com TPR e Grupo 3: controle positivo recebeu monensina sódica na dose de 30 gramas por tonelada). * Colunas seguidas de letras iguais e/ou sem identificação não diferem entre si.

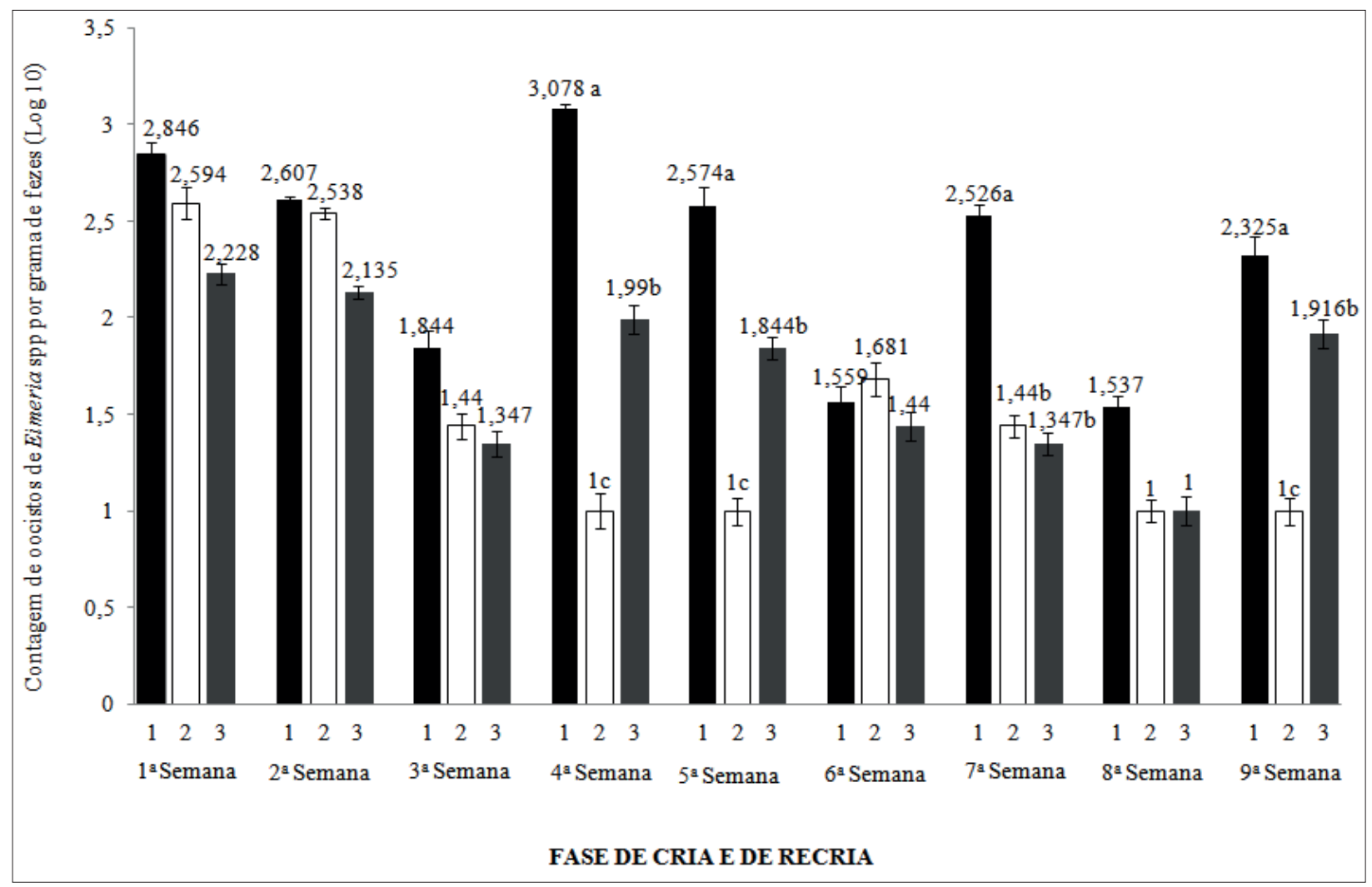

Fonte: Elaboração dos autores.

\section{Conclusão}

Observou-se que a torta de pimenta rosa adicionada a dieta de caprinos foi eficaz no controle de protozoários da família Eimeriidae, com potencial para serem utilizadas em programas alternativos de controle parasitário, além de corroborar no desempenho animal conferindo melhor ganho de peso.

\section{Referências}

AFONSO, J. A. B.; MENDONÇA, C. L.; FIORAVANTE,
M. C. S.; KUCHEMBUCK, M. R. G. Características e indicações clínicas dos ionóforos para ruminantes. Revista do CFMV, Brasília, v. 20, n. 20, p. 29-3, 2000.

AGAOGLU, Z. T.; AKGUL, Y.; KELES, I.; UGRAS, S.; AKSOY, A.; CINAR, A. Accidental salynomycin intoxication of Angora goats in Turkey. Small Ruminant Research, Amsterdam, v. 45, n. 2, p. 159-161, 2002.

ALEMÁN, Y.; SÁNCHEZ, L. M.; PÉREZ, T.; RODRÍGUEZ, Y.; OLIVARES, J. L; RODRÍGUEZ, J. G. Actividad larvicida de extractos de Rhizophora mangle L. contra estrongílidos gastrointestinales de ovinos. Revista de Salud Animal, Cuba, v. 33, n. 2, p. 111-115, 2011. 
ALMEIDA, M. A. O.; DOMINGUES, L. F.; ALMEIDA, G. N.; SIMAS, M. M. S.; BOTURA, M. B.; CRUZ, A. C. F. G.; SILVA, A. V. A. F.; MENESES, T. P.; BATATINHA, M. J. M. Efeitos dos extratos de folhas de Mentha piperita L. e de Chenopodium ambrosoides L. sobre Cultivos de larvas infectantes de nematóides gastrintestinais de caprinos. Brazilian Journal of Veterinary Parasitology, Jaboticabal, v. 16, n. 1, p. 57-59, 2007.

ATHANASIADOU, S.; HOUDIJK, J.; KYRIAZAKIS, I. Exploiting synergisms and interactions in the nutritional approaches to parasite control in sheep production systems. Small Ruminant Research, Amsterdam, v. 76, n. 1, p. 2-11, 2008.

ATHANASIADOU, S.; KYRIAZAKIS, I.; JACKSON, F.; COOP, R. L. Direct anthelmintic effects of condensed tannins towards different gastrointestinal nematodes of sheep: in vitro and in vivo studies. Veterinary Parasitology, Amsterdam, v. 99, n. 3, p. 205-219, 2001.

BARROS, N. N.; SANTOS, Y. C. C.; FERREIRA, M. P. B. Uso de promotores de crescimento para cabritos leiteiros, na fase de recria. Revista Científica de Produção Animal, Teresina, v. 1, n. 1, p. 9-16, 1999.

BARRY, T. N.; MCNEILL, D.; MCNABB, W. C. Plant secondary compounds: their impact on forage nutritive value and upon animal production. In: INTERNATIONAL GRASSLAND CONGRESS, 19., 2001, São Pedro. Proceedings... Piracicaba: FEALQ, 2001. p. 445-52.

BERGEN, W. G.; BATES, D. B. Ionophores: their effect on production efficiency and mode of action. Journal of Animal Science, Champaign, v. 58, n. 6, p. 1465, 1984.

CEZAR, A. S.; CATTO, J. B.; BIANCHIN, I. Controle alternativo de nematódeos gastrintestinais dos ruminantes: atualidade e perspectivas. Ciência Rural, Santa Maria, v. 38, v. 7, p. 2083-2091, 2008.

COSTA, V. M. M.; SIMOES, S. V. D.; RIET-CORREA, F. Doenças parasitárias em ruminantes no semi-árido brasileiro. Pesquisa Veterinária Brasileira, Seropédica, v. 29 , n. 7 , p. 563-568, 2009.

DAWRA, R. K.; MAKKAR, H. P. S.; SINGH, B. Proteinbinding capacity of microquantities of tannins. Analytical Biochemistry, Philadelphia, v. 170, n. 1, p. 50-53, 1988.

FALBO, M. K.; SANDINI, I. E.; ISHIY, H. M.; FÁVARO, J. L.; SANTOS, C. D.; FALBO, S.; RODIGHERI, D.; GUZZO, D. Atividade Anti-Helmíntica do fruto da Melia azedarach em cordeiros naturalmente infectados com nematódeos gastrintestinais. Semina: Ciências Agrárias, Londrina, v. 29, n. 4, p. 881-886, 2008.

FORBES, A. B.; CUTLER, K. L.; RICE, B. J. Sub- clinical parasitism in spring-born, beef suckler calves: epidemiology and impact on growth performance during the first grazing season. Veterinary Parasitology, Amsterdam, v. 104, n. 4, p. 339-344, 2002.

FREITAS, F. L. C.; ALMEIDA, K. S.; NASCIMENTO, A. A.; MACHADO, C. R.; VESCHI, J. L. A.; MACHADO, R. Z. Espécies do gênero Eimeria Schneider, 1875 (Apicomplexa: Eimeriidae) em caprinos leiteiros mantidos em sistema intensivo na região de São José do Rio Preto, estado de São Paulo, Brasil. Revista Brasileira de Parasitologia Veterinária, São Carlos, v. 14, n. 1, p. 7-10, 2005.

GAZZANEO, L. R. S.; LUCENA, R. F. P.; ALBUQUERQUE, U. P. Knowledge and use of medicinal plants by local specialists in a region of Atlantic Forest in the state of Pernambuco (Northeastern Brazil). Journal of Ethnobiology and Ethnomedicine, London, v. 32, n. 1, p. 1-11, 2005.

GITHIORI, J. B.; ATHANASIADOU, S.; THAMSBORG, S. M. Use of plants in novel approaches for control of gastrointestinal helminths in livestock with emphasis on small ruminants. Veterinary Parasitology, Amsterdam, v. 139, n. 4, p. 308-320, 2006.

GOMES, R. V. R. S.; VILELA, V. L. R.; GOMES, E. N.; MAIA, A. J.; ATHAYDE, A. C. R. Ação antiparasitária in vitro dos extratos etanólicos de Operculina hamiltonii (batata de purga) e Momordica charantia (melão de são caetano) sobre ovos e larvas de nematóides gastrintestinais de caprinos do semi-árido paraibano. Acta Veterinaria Brasilica, Mossoró, v. 4, n. 2, p. 92-99, 2010.

GONÇALVES, F. G.; ZANINI, S. F.; FEITOSA, M. L.; GONÇALVES, E. P. M.; COLNAGO, G. L. Efeito da pimenta rosa associada a diversas dosagens de antibióticos em frangos de corte. Ciência Rural, Santa Maria, v. 42, n. 8, p. 1503-1509, 2012.

GORDON, H. M.; WHITLOCK, H. V. A new technique for courting nematode eggs in sheep faeces. Journal of Council of Science and Industry Research, Melbourne, v. 12, n. 1, p. 50-52, 1939.

GRAMINHA, E. B. N.; MONTEIRO, A. C.; SILVA, H. C.; OliveIRA, G. P.; COSTA, A. J. Controle de Nematóides parasitos gastrintestinais de ovinos naturalmente infestados mantidos em pastagens. Pesquisa Agropecuária Brasileira, Brasília, v.40, n. 9, p. 927-933, 2005.

HECKENDORN, F.; HÄRING, D. A.; MAURER, V.; SENN, M.; HERTZBERG, H. Individual administration of three tanniferous forage plants to lambs artificially infected with Haemonchus contortus and Cooperia curticei. Veterinary Parasitology, Amsterdam, v. 146, n. 
1-2, p. 123-134, 2007.

HOSTE, H.; JACKSON, F.; ATHANASIADOU, S.; THAMSBORG, S. M.; HOSKIN, S. O. The effects of tannin-rich plants on parasitic nematodes in ruminants. Trends in Parasitology, Philadelphia, v. 22, n. 6, p. 253261, 2006.

HOSTE, H.; TORRES-ACOSTA, J. F.; PAOLINI, V.; AGUILAR-CABALLERO, A.; ETTER, E.; LEFRILEUX, Y.; CHARTIER, C.; BROQUA, C. Interactions between nutrition and gastrointestinal infections with parasitic nematodes in goats. Small Ruminant Research, Amsterdam, v. 60, n. 1-2, p. 141$151,2005$.

INOUE, K. H.; HAGERMAN, A. E. Determination of gallotannin with rhodanine. Analytical Biochemistry. v. 169, n. 2, p. 363-369. 1988.

JOSHI, B. R.; KOMMURU, D. S.; TERRILL, T. H.; MOSJIDIS, J. A.; BURKE, J. M.; SHAKYA, K. P.; MILLER, J. E. Effect of feeding sericea lespedeza leaf meal in goats experimentally infected with Haemonchus contortus. Veterinary Parasitology, Amsterdam, v. 178, n. 1-2, p. 192-197, 2011.

KOTTEK, M.; GRIESER, J.; BECK, C.; RUDOLF, B.; RUBEL, F. World map of the köppen-geiger climate classification updated. Meteorologische Zeitschrift, Viena, v. 15, n. 3, p. 259-263, 2006.

LEVINE, N. D. Veterinary protozoology. Ames: Iowa State University Press, 1985. 414 p.

LIMA, M. R. F.; XIMENES, E. C. P. A.; LUNA, J. S.; SANT'ANA, A. E. G. The antibiotic activity of some Brazilian medicinal plants. Revista Brasileira de Farmacognosia, Curitiba, v. 16, n. 3, p. 300-306, 2006.

LIMA, V. X. M.; AHID, S. M. M.; SIMPLICIO, A. A. Efeito de sal mineral enriquecido ou não com ionóforos sobre a frequência de eimerí́deos de fêmeas caprinas jovens. Revista em Agronegócio e Meio Ambiente, Maringá, v. 2, n. 2, p. 63-71, 2009.

MAKKAR, H. P. S.; BLÜMMEL, M.; BOROWY, N. K.; BECKER, K. Gravimetric determination of tannins and their correlation with chemical and protein precipitation methods. Journal of the Science of Food and Agriculture, Hoboken, v. 61, n. 2, p. 161-165, 1993.

MARTINEZ, M. J.; GONZALES, N. A.; BETNCOURT, J.B. Actividad antimicrobiana del Schinus terebinthifolius Raddi (copal). Revista Cubana de Plantas Medicinales, Cuba, v. 1, n. 3, p. 37-9, 1996.

MAX, R. A.; KASSUKU, A. A.; KIMAMBO, A. E.; MTENGA, L. A.; WAKELIN, D.; BUTTERY, J. The effect of wattle tannin drenches on gastrointestinal nematodes of tropical sheep and goats during experimental and natural infections. Journal of Agricultural Science, Cambridge, v. 147, n. 2, p. 211-218, 2009.

MIN, B. R.; HART, S. P. Tannins for suppression of internal parasites. Journal of Animal Science, Champaign, v. 81, n. 14, p. 102-109, 2003. Suplemento 2.

MIN, B. R.; HART, S. P.; MILLER, D.; TOMITA, G.; LOETZ, E.; SAHLU, T. The effect of grazing forage containing condensed tannins on gastro-intestinal parasite infection in Angora does. Veterinary Parasitology, Amsterdam, v. 130, n. 1-2, p. 105-13, 2005.

MIN, B. R.; MILLER, D.; HART, S. P.; TOMITA, G.; LOETZ, E.; SAHLU, T. Direct effects of condensed tannins on gastrointestinal nematodes in grazing Angora goats. Journal of Animal Science, Champaign, v. 81 (Suppl. 2):23, 2003.

MINHO, A. P.; BUENO, I. C. S.; GENNARI, S. M.; JACKSON, F.; ABDALLA, A. L. In vitro effect of condensed tannin extract from acacia (Acacia mearnsii) on gastrointestinal nematodes of sheep. Revista Brasileira de Parasitologia Veterinária, São Carlos, v. 17, (Suppl. 2): 144-148, 2008b.

MINHO, A. P.; LOUVANDINI, H.; JACKSON, F.; BUENO, I. C. S.; GENNARI, S. M.; ABDALLA, A. L. Effect of Acacia molissima tannin extract on the control of gastrointestinal parasites in sheep. Animal Feeding Scince Technology, v. 147, n. 1-3, p. 172-181, 2008a.

MUPEYO, B.; BARRY, T. N.; POMROY, W. E.; RAMIREZ-RESTREPO, C. A.; LOPEZ-VILLALOBOS, N.; PERNTHANER, A. Effects of feeding willow (Salix spp.) upon death of established parasites and parasite fecundity. Animal Feed Science and Technology, v. 164, n. 1-2, p. 8-20, 2011.

NAGARAJA, T. G.; AVERY, T. B.; BARTLEY, E. E.; GALITZER, S. J.; DAYTON, A. D. Prevention of lactic acidosis in cattle by lasolocid or monensin. Journal of Animal Science, Champaign, v. 53, n. 1, p. 206-216, 1981.

NASCIMENTO, T. V. C.; NOGUEIRA, D. M.; VOLTOLINI, T. V.; CAVALCANTI, N. B. Desempenho produtivo e controle de nematódeos gastrintestinais em caprinos suplementados com frutos do umbuzeiro na Região Semiárida. Revista Brasileira de Agroecologia, v. 4, n. 2, p. 2855-2858, 2009.

NOGUEIRA, F. A.; OLIVEIRA, L. N.; SILVA, R. B. da; NERY, P. S.; VIRGÍNIO JUNIOR, G. F.; GERASEEV, L. C.; DUARTE, E. R. Anthelminthic efficacy of banana crop residues on gastrointestinal nematodes of sheep: in vitro and in vivo tests. Parasitology Research, v. 111, n. 1, p. 317-323, 2012. 
OLIVEIRA, L. M. B.; BEVILAQUA, C. M. L.; MORAIS, S. M. de; CAMURÇA-VASCONCELOS, A. L. F.; MACEDO, I. T. F. Plantas taniníferas e o controle de nematóides gastrintestinais de pequenos ruminantes. Ciência Rural, Santa Maria, v. 41, n. 11, p. 1967-1974, 2011.

OLIVEIRA, L. N.; DUARTE, E. R.; NOGUEIRA, F. A.; SILVA, R. B. da; FARIA FILHO, D. E. de; GERASEEV, L. C. Eficácia de resíduos da bananicultura sobre a inibição do desenvolvimento larval em Haemonchus spp. provenientes de ovinos. Ciência Rural, Santa Maria, v. 40, n. 2, p. 458-460, 2010.

OLIVEIRA, P. R.; LIMA, J. D.; SILVA, A. C.; LABRUNA, M. B.; FACURY FILHO, E. J. Controle da infecção por Eimeria spp., em caprinos pela administração contínua de salinomicina no suplemento mineral. Arquivo Brasileiro de Medicina Veterinária e Zootecnia, v. 49, n. 3, p. 291-296, 1997.

OTERO, M. J.; HIDALGO, L. G. Taninos condensados en espécies forrajeras de clima templado: efectos sobre productividad de rumiantes afectados por parasitosis gastrointestinales. Livestock Research for Rural Development, v. 16, n. 2, p. 1-9, 2004.

PARRA, C. L. C.; OLIVO, C. J.; FLORES, F. S.; AGNOLIN, C. A.; PIRES, C. C.; BOLZAN, A. M. Alteração da carga de endoparasitas em ovinos submetidos a diferentes níveis de folha de bananeira na alimentação. Revista Brasileira de Agroecologia, v. 6, n. 2, p. 111-116, 2011.

PORTER, L. J.; HRSTICH, L. N.; CHAN, B. G. The conversion of procyanidins and prodelphinidins to cyanidin and delphinidin. Phytochemistry, v. 25, n. 1, p. 223-230, 1985.

RUSSEL, J. B.; STROBEL, H. J. Effects os additives on in vitro ruminal fermentation: a comparison of monensin and bacitracin, another gram-positive antibiotic. Journal of Animal Science, Champaign, v. 66, n. 2, p. 552-558, 1988.

SANTA-ROSA, J.; PINHEIRO, P. A. Intoxicação por salinomicina em cabritos. In: CONGRESSO BRASILEIRO DE MEDICINA VETERINÁRIA, 24., 1996, Goiânia. Anais... Goiânia: Sociedade Pernambucana de Medicina Veterinária, 1996. p. 60.

SHAIK, S. A.; TERRILL, T. H.; MILLER, J. E.; KOUAKOU, B.; KANNAN, G.; KAPLAN, R. M.; BURKE, J. M.; MOSJIDIS, J. A. Sericea lespedeza hay as a natural deworming agent against gastrointestinal nematode infection in goats. Veterinary Parasitology, Amsterdam, v. 139, n. 1-3, p. 150-157, 2006.

SILVA, C. F.; ATHAYDE, A. C. R.; SILVA, W. W.;
RODRIGUES, O. G.; VILELA, V. L. R.; MARINHO, P. V. T. Avaliação da eficácia de taboa (Typha domingensis Pers.) e batata-de-purga [Operculina hamiltonii (G. Don) D.F. Austin \& Staples] in natura sobre nematóides gastrintestinais de caprinos, naturalmente infectados, em clima semi-árido. Revista Brasileira de Plantas Medicinais, Botucatu, v. 12, n. 4, p. 466-471, 2010.

SILVA, D. J. Análise de alimentos: métodos químicos e biológicos. 2. ed. Viçosa. Editora UFV, 1998. 165 p.

SIMPLÍCIO, A. A.; CASTELO, T. S.; SILVA, A. R.; MOTA-FILHO, A. C.; COSTA, L. L. M. Parâmetros reprodutivos de fêmeas caprinas jovens suplementadas com sal mineral adicionado ou não com ionóforos. Acta Veterinaria Brasilica, Mossoró, v. 5, n. 3, p. 259-263, 2011.

STOCK, R.; MADER, T. Feed additives for beef cattle. Nebguide, 1997. G85-761-A. Disponível em: <http:// www.ianr.unl.edu/pubs/ beef/g761.htm>. Acesso em: 05 out. 2010.

TERRIL, T. H.; MOSJIDIS, J. A.; MOORE, D. A.; SHAIK, S. A.; MILLER, J. E.; BURKE, J. M.; MUIR, J. P.; WOLFE, R. Effect of pelleting on efficacy of sericea lespedeza hay as natural dewormer in goats. Veterinary Parasitology, Amsterdam, v. 146, n. 1-2, p. 117-122, 2007.

VASTA, V.; MELE, M.; SERRA, A.; SCERRA, M.; LUCIANO, G.; LANZA, M.; PRIOLO, A. Metabolic fate of fatty acids involved in ruminal biohydrogenation in sheep fed concentrate or herbage with or without tannins. Journal of Animal Science, Champaign, v. 87, n. 8, p. 2674-2684, 2009.

VIEIRA, L. S. Métodos alternativos de controle de nematóides gastrintestinais em caprinos e ovinos. Tecnologia e Ciência Agropecuária, João Pessoa, v. 2, n. 2, p. 49-56, 2008.

VIEIRA, L. S.; BARROS, N. N.; CAVALCANTE, A. C. R.; XIMENES, L. J. F.; CARVALHO, R. B. A salinomicina para o controle da eimeriose de caprinos leiteiros nas fases de cria e recria. Ciência Rural, Santa Maria, v. 34, n. 3, p. 873-878. 2004.

VIEIRA, L. S.; LOBO, R. N. B.; BARROS, N. N.; PORTELA, C. H. P.; SIMPLÍCIO, A. A. Monensina sódica no controle da eimeriose em caprinos leiteiros. Ciência Animal, Santa Maria, v. 15, n. 1, p. 25-31, 2005.

VIEIRA, L. S.; CAVALCANTE, A. C. R.; PEREIRA, M. F.; DANTAS, L. B.; XIMENES, L. J. F. Evaluation of anthelmintic efficacy of plants available in Ceará State, North - East Brazil, for the control of goat gastrointestinal nematodes. Revue de Médecine Vétérinaire, Toulouse, v. 150, n. 5, p. 447-52, 1999. 
ZANINI, S. F.; COLNAGO, G. L.; GONÇALVES, F. G.; AZEVEDO, M. A. S., VENIAL, H. J.; SOUSA, D. R.; MUSSI, J. M. S.; SILVA, M. A. Uso de salvado de aroeiraroja (Schinus terebinthifolius Raddi) desengordurado en la dieta de pollos de engorde. In: CONGRESSO LATINOAMERICANO DE AVICULTURA, 11., 2009, Cuba. Anais...Cuba: ALA, 2009a. 1 CD-ROM.
ZANINI, S. F.; COLNAGO, G. L.; GONÇALVES F. G.; FEITOSA, M. L.; AZEVEDO, M. A. S., VENIAL, H. J.; MUSSI, J. M. S. Avaliação da eficiência do farelo bruto de aroeira-vermelha (Schinus terebinthifolius Raddi) na dieta de frangos de corte como agente anticoccidiano. In: CONGRESSO BRASILEIRO DE MEDICINA VETERINÁRIA, 36., 2009, Porto Seguro. Anais... Porto Seguro: SBMV, 2009b. 1 CD-ROM. 
